\title{
A multiplex PCR test to identify four common cattle-adapted Cryptosporidium species
}

\author{
SARAH THOMSON ${ }^{1}$, ELISABETH A. INNES ${ }^{1}$, NICHOLAS N. JONSSON ${ }^{2}$ and \\ FRANK KATZER ${ }^{1} *$ \\ ${ }^{1}$ Moredun Research Institute, Pentlands Science Park, Bush Loan, Edinburgh EH26 OPZ, UK \\ ${ }^{2}$ College of Medical, Veterinary and Life Sciences, University of Glasgow, 464 Bearsden Road, Glasgow G61 1QH, UK
}

(Received 10 December 2015; revised 25 February 2016; accepted 12 March 2016)

\section{SUMMARY}

Cryptosporidium is a well-known cause of neonatal enteritis in cattle worldwide. Cattle are commonly infected with four different species of Cryptosporidium but only one of these, Cryptosporidium parvum, is associated with clinical disease. Identification of species in cases of calf scour can give an indication if Cryptosporidium is the causative agent or not. In addition, $C$.parvum is a zoonotic species and so has implications for human health, for this reason it is important to identify the species of Cryptosporidium infecting cattle particularly where a farm is implicated in an outbreak of cryptosporidiosis in humans. Here a multiplex PCR test, which can identify the four common cattle-adapted Cryptosporidium species, including C.parvum, has been developed. This test allows quick and accurate detection of Cryptosporidium species in cattle fecal samples including mixed infections, which could be missed by the more common method of sequencing the same gene.

Key words: Cryptosporidium, cattle, detection, multiplex, PCR.

\section{INTRODUCTION}

Cryptosporidiosis is the disease caused by the protozoan parasite Cryptosporidium, which affects many different species of mammals, including humans, birds, reptiles, amphibians and fish (Fayer, 2010; Chalmers and Katzer, 2013; Ryan et al. 2014). It has been identified as one of the most important causes of bovine neonatal enteritis worldwide (De Graaf et al. 1999; Brook et al. 2008; Silverlas et al. 2009; Wyatt et al. 2010).

The disease usually manifests itself as profuse watery diarrhoea, loss of appetite and abdominal pain (Clark and Sears, 1996; Tzipori and Ward, 2002; Klein et al. 2008) which lasts approximately 2 weeks with little or no long-term implications on animal health (Klein et al. 2008). Most animals will recover but in severe cases complications may arise and death can occur. There are currently over 27 recognized species of Cryptosporidium, four of which commonly infect cattle; Cryptosporidium parvum, Cryptosporidium andersoni, Cryptosporidium bovis and Cryptosporidium ryanae (Chalmers and Katzer, 2013; Ryan et al. 2014).

Detection of Cryptosporidium in farm livestock is usually done by microscopy (in veterinary diagnostic laboratories) or by molecular detection (research laboratories); of the two methods molecular detection is much more sensitive and allows full speciation (Chalmers and Katzer, 2013). One of the most common genes targeted for PCR detection of

* Corresponding author. Moredun Research Institute, Pentlands Science Park, Bush Loan, Edinburgh, EH26 OPZ, UK. Tel.: +44 (0)31 445 5111. Fax: +44 (0)131 445 6111. E-mail: frank.katzer@moredun.ac.uk
Cryptosporidium is the $18 \mathrm{~S}$ rRNA gene, which is located on a multi-copy DNA element meaning that the PCR is more sensitive than those which target a single copy gene. The $18 \mathrm{~S}$ rRNA gene is also one of the most commonly used genes for species discrimination and there are many $18 \mathrm{~S}$ rRNA reference sequences available on the National Centre for Biotechnology Information website (www.ncbi.nlm.nih.gov). In order to identify species in Cryptosporidium-positive samples, it is necessary to carry out sequencing or PCRRFLP (polymerase chain reaction followed by restriction fragment length polymorphism). These methods can be expensive, time consuming and it is very difficult to detect mixed infections as often only the most predominant species in a sample is detected (Cama et al. 2006). PCR-RFLP can also detect only a limited number of species.

A multiplex PCR test would enable the rapid speciation of a subset of Cryptosporidium species and previous attempts have been made to develop such a test (Patel et al. 1999; Lindergard et al. 2003; Santin and Zarlenga, 2009); the most recent of which can detect the four most commonly found Cryptosporidium species in cattle (Santin and Zarlenga, 2009). This test cannot distinguish between $C$. bovis and $C$. ryanae and a further PCR amplification and sequencing would be required to distinguish these species.

In this paper, we describe the development of a multiplex PCR test to identify three cattle-adapted species (C. andersoni, C. bovis and C. ryanae) plus the zoonotic species $C$. parvum in a nested PCR. It is also possible with this multiplex PCR to detect other Cryptosporidium species by sequencing of the

Parasitology Open (2016), Vol. 2, e5; page 1 of 9. (C) Cambridge University Press 2016. This is an Open Access article, distributed under the terms of the Creative Commons Attribution licence (http://creativecommons.org/licenses/by/4.0/), which permits unrestricted re-use, distribution, and reproduction in any medium, provided the original work is properly cited. 
18S rRNA gene, where none of the other species have been detected. This PCR test will be extremely useful in veterinary diagnostics as a tool for accurate diagnosis of scour in neonatal calves and as a research tool to investigate the epidemiology of cryptosporidiosis. The nssm-PCR would allow speciation but would not permit sub-typing, which would be necessary for outbreak investigation. Current diagnostic methods can only confirm that Cryptosporidium spp. are present in the feces, but cannot distinguish the disease causing $C$. parvum species from the less virulent $C$. andersoni which has been associated with a reduction in weight-gain and milk yield in adult cattle, $C$. bovis and $C$. ryanae, which have not yet been associated with the clinical disease. The test is already being used to monitor calves on a veterinary school farm to ensure that the calves used for practical classes are free from $C$. parvum to minimize the risk to the students.

\section{MATERIALS AND METHODS}

\section{Design of Cryptosporidium species-specific primers}

Alignments of multiple 18S rRNA gene sequences (between 50 and 100 for each species) for Cryptosporidium species (C. parvum, C. bovis, $C$. ryanae, C. andersoni, C. ubiquitum, C. hominis, $C$. muris, $C$. xiaoi and C. suis) were made using the BioEdit Software Program (www.mbio.ncsu.edu/ bioedit/bioedit.html) to identify representative sequences for each species. Clustal W (www.ebi.ac. uk/Tools/msa/clustalw2) alignments of the representative sequences were made and regions for speciesspecific primers were identified which would produce amplicons of different sizes for each species when used with the 18S rRNA reverse primer (AL3032) (Xiao et al. 2000). The locations of the primers used are shown in Fig. 1; The outer primers are the same as those described by Xiao et al. 2000 (AL1691/ AL1687) as are the first set of inner primers (AL1598 and AL3032) these amplify a $840 \mathrm{bp}$ genus-specific fragment, the second inner primer set $(\mathrm{CaF}$ and AL3032) amplify a 625 bp fragment from $C$. andersoni; the third set ( $\mathrm{CrF}$ and AL3032) amplify a $415 \mathrm{bp}$ fragment from $C$. ryanae the forward primer in this set contains a degenerate base $(\mathrm{R})$ where the $C$. ryanae sequence contains either an ' $A$ ' or a ' $G$ '; the fourth set (CphF and AL3032) amplify a $305 \mathrm{bp}$ fragment from C. parvum, C. hominis and C. suis, which can be later distinguished with an additional PCR if desired and the final set of primers (CbF and AL3032) amplify a $241 \mathrm{bp}$ fragment from $C$. bovis. The primer sequences are shown in Table 1.

\section{Fecal sample collection and DNA extraction}

Fecal samples $(n=498)$ were collected from 190 calves and 308 adult cattle from 21 beef farms as part of another study. These samples were collected from calves aged 7-14 days regardless of clinical signs $(n=116)$, all calves suffering from diarrhoea regardless of age $(n=74)$, adult cattle pre- and post-calving ( $n=177$ and 120 , respectively) and other adult cows $(n=11)$. Samples were collected by the farmers and sent to the Moredun Research Institute by their veterinary practices.

DNA was extracted from the calf feces processing with a QIAamp DNA Stool Minikit (Qiagen) according to the manufacturer's instructions except that $10 \times$ freeze-thaw cycles in liquid nitrogen were carried out after adding $200 \mu \mathrm{g}$ sample to lysis buffer (provided in the kit). Adult feces were subjected to a sedimentation step prior to DNA extraction. The resultant pellet was added to lysis buffer and subjected to $10 \times$ freeze-thaw cycles in liquid nitrogen followed by processing by QIAamp DNA Stool Minikit (Qiagen) according to the manufacturer's instructions.

To identify Cryptosporidium-positive samples $18 \mathrm{~S}$ nested PCR was carried out as described by Xiao et al. 2000. This work was carried out as part of another study at the Moredun Research Institute. Microscopic examination of the feces was not carried out for any of the samples.

\section{Testing of individual species-specific primers}

First round reactions were carried out as described by Xiao et al. 2000 using DNA from known positive samples for each of the four species. Secondary reactions were carried out to test each individual speciesspecific forward primer with the reverse $18 \mathrm{~S}$ rRNA primer (AL3032). First round PCR products were

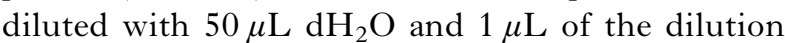
used as a template in a reaction containing $2.5 \mu \mathrm{L}$ 10× PCR buffer (45 mu Tris-HCL pH 8.8, $11 \mathrm{~mm}$ $\left(\mathrm{NH}_{4}\right)_{2} \mathrm{SO}_{4}, 4.5 \mathrm{~mm} \mathrm{MgCl}_{2}, 4.4 \mu \mathrm{M} \mathrm{EDTA}, 113 \mu \mathrm{g}$ $\mathrm{mL}^{-1} \mathrm{BSA}, 1 \mathrm{mM}$ of each of the four deoxyribonucleotide triphosphates) (Katzer et al. 2014), 0.5 unit Bio'Taq (Bioline, UK) and $10 \mu \mathrm{M}$ forward and reverse primers. The final volume of each reaction was made up to $25 \mu \mathrm{L}$ using $\mathrm{dH}_{2} \mathrm{O}$.

Cycling conditions were $3 \mathrm{~min}$ at $94{ }^{\circ} \mathrm{C}$, followed by 35 cycles of $45 \mathrm{~s}$ at $94^{\circ} \mathrm{C}, 45 \mathrm{~s}$ on a temperature gradient of $49-59^{\circ} \mathrm{C}$ and $1 \mathrm{~min}$ at $72{ }^{\circ} \mathrm{C}$. The final extension was $7 \mathrm{~min}$ at $72^{\circ} \mathrm{C}$. Second round PCR products were visualized following electrophoresis on a $1 \cdot 5 \%$ agarose gel stained with GelRed ${ }^{\mathrm{TM}}$ (Biotium, UK) on an AlphaImager 2200.

\section{Field samples}

Primers were tested on a panel of the only available DNA samples, which included known positive samples for C. parvum, C. andersoni, C. bovis and $C$. ryanae from naturally infected cattle aged from 1 day old to adult animals $(n=128)$. Results from 

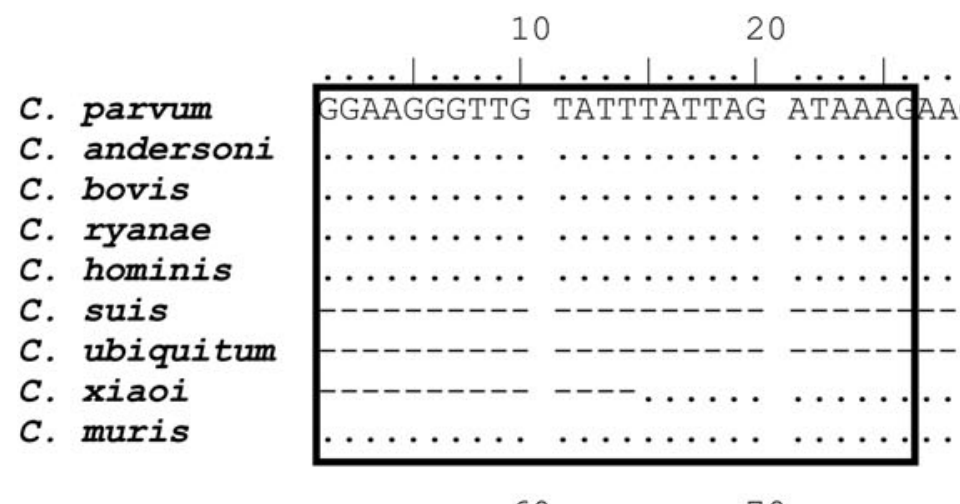

30

40

60

70

80

$\ldots|\ldots| \ldots|\ldots|$

AACC AATATAATT- -GGTGACTCA

...GAGC. . - ....T. . .

.G..TT.. - - . . W.

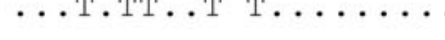

C. parvum

$\ldots|\ldots| \ldots|\ldots| \ldots|\ldots| \ldots|\ldots| \ldots|\ldots| \ldots \mid$

C. andersoni

TAATAACTTT ACGGATCACA TTAA--ATGT GACATATCAT TCAAGTTTCT

C. bovis

C. ryanae

C. hominis

C. suis

C. ubiquitum

C. xiaoi

C. muris

$\begin{array}{rrrrr}110 & 120 & 130 & 140 & 150\end{array}$
C. parvum
C. andersoni
c. bovis
C. ryanae
C. hominis
C. suis
C. ubiquitum
C. xiaoi
C. muris

140

.1

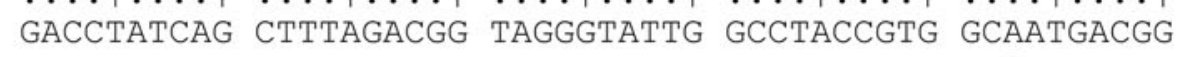

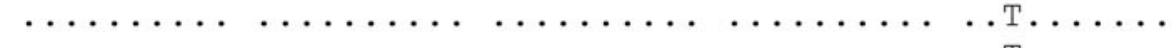

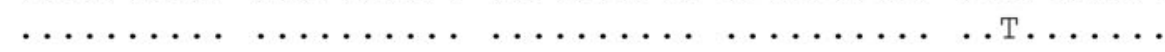

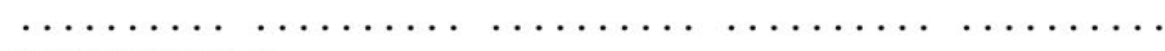

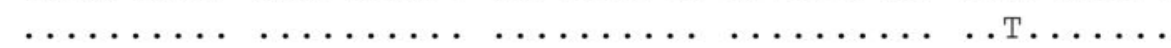$$
\ldots \ldots \ldots \ldots \ldots \ldots \ldots \ldots \ldots \ldots \ldots \ldots \ldots
$$

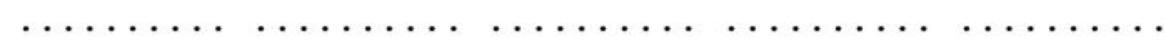

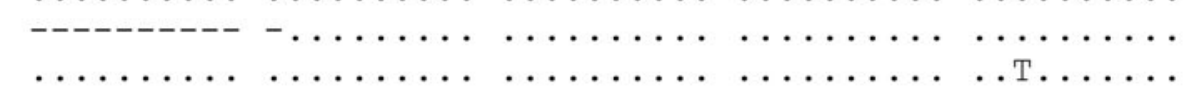

$\begin{array}{lllll}160 & 170 & 180 & 190 & 200\end{array}$
C. parvum
C. andersoni
C. bovis
c. ryanae
c. hominis
C. suis
C. ubiquitum
c. xiaoi
c. muris 160

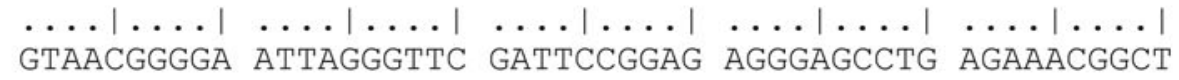

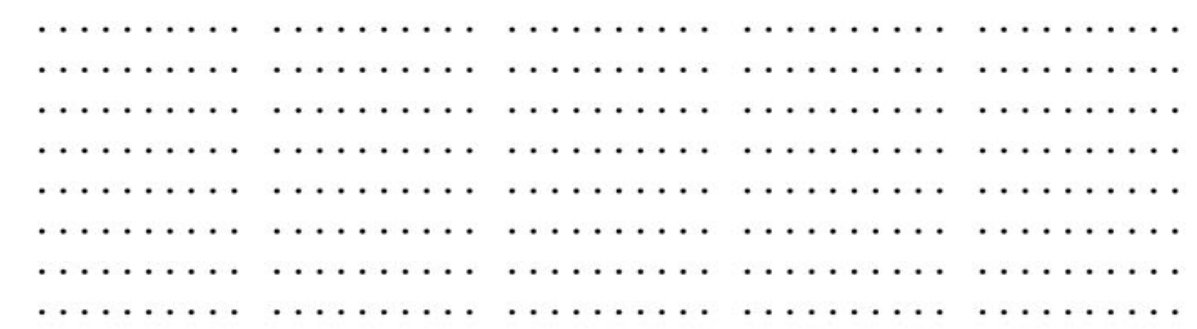

$\begin{array}{rrrrr}210 & 220 & 230 & 240 & 250\end{array}$
C. parvum
C. andersoni
c. bovis
c. ryanae
C. hominis
C. suis
C. ubiquitum
C. xiaoi
C. muris

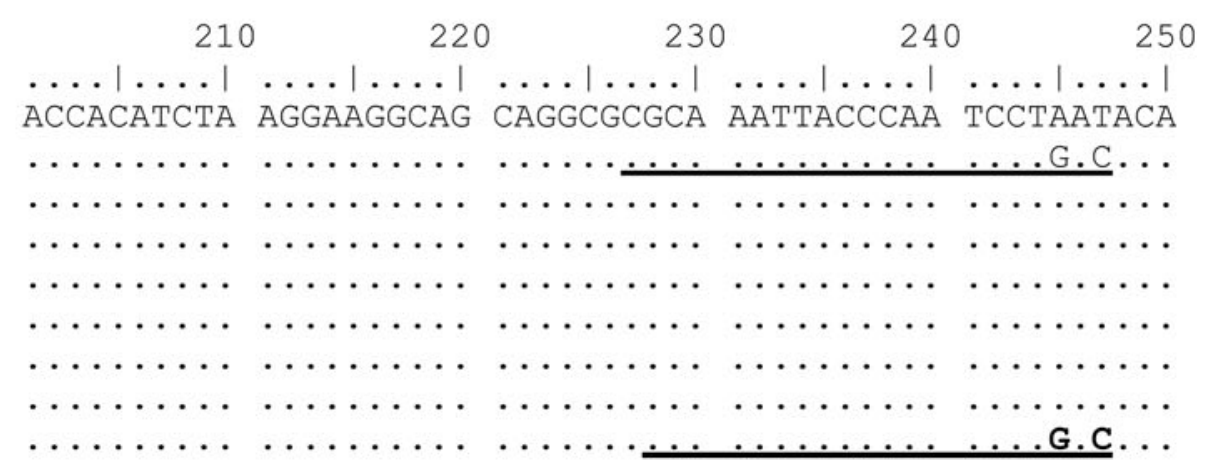

Fig. 1. Positions of species-specific primers on the $18 \mathrm{~S}$ rRNA gene plus the location of the $18 \mathrm{~S}$ rRNA internal primers. 

C. parvum
C. andersoni
C. bovis
C. ryanae
C. hominis
C. suis
C. ubiquitum
C. xiaoi
C. muris

260

270

280

290

300

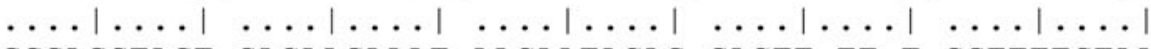

$$
\ldots . . .
$$

$\cdots$

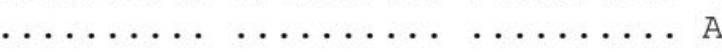

$\cdots \cdots \cdots$

$\begin{array}{llll}310 & 320 & 330 & 340\end{array}$
C. parvum
C. andersoni
C. bovis
C. ryanae
C. hominis
C. suis
C. ubiquitum
c. xiaoi
C. muris
C. parvum
C. andersoni
C. bovis
C. ryanae
C. hominis
C. suis
C. ubiquitum
C. xiaoi
C. muris
C. parvum
C. andersoni
C. bovis
C. ryanae
C. hominis
C. suis
C. ubiquitum
C. xiaoi
C. muris

410

420

430

440

450

$\ldots|\ldots| \ldots|\ldots| \ldots|\ldots| \ldots|\ldots| \ldots|\ldots| \ldots|\ldots| \ldots|\ldots| \ldots|\ldots| \ldots \mid$ GTTGTTGCAG TTAAAAAGCT CGTAGTTGGA TTTCTGTTAA TAATTTATAT
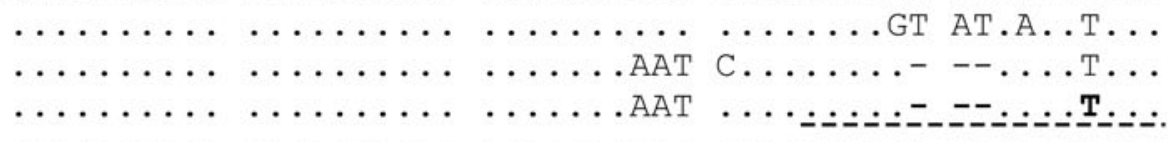

..... . . . . . . . . . . . . . . . . . . . . . . . . . . .

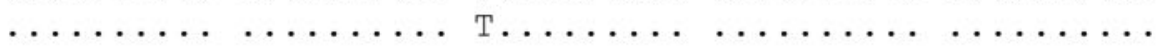

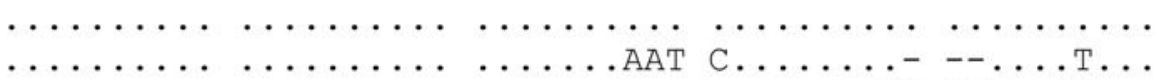

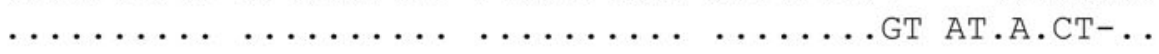
C. parvum
C. andersoni
C. bovis
C. ryanae
C. hominis
C. suis
C. ubiquitum
C. xiaoi
C. muris

460

470

480

490

500

$$
\ldots|\ldots| \ldots|\ldots| \ldots|\ldots| \ldots|\ldots| \ldots|\ldots| \ldots \mid
$$

AAAATATTTT GA-TGAATAT TTAT-ATAAT ATTAACATAA TTCATATTAC

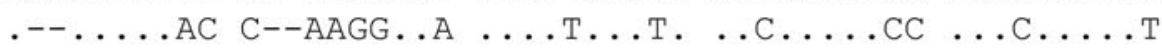

.T- ..A.A. C--ACG.

IT-. C.A.GC T- T-ACGG.

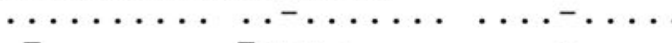

$. \mathrm{T} \ldots \ldots \mathrm{T}---\ldots \ldots \ldots$

. T...... ATT--.......... .

.T-.C.A.A. C--ACG...........

.$-\ldots .$. AC T--AAGG... A... T...

Fig. 1. Continued 

C. parvum
C. andersoni
C. bovis
C. ryanae
C. hominis
C. suis
C. ubiquitum
C. xiaoi
C. muris $\ldots|\ldots| \ldots|\ldots| \ldots|\ldots| \ldots|\ldots| \ldots|\ldots| \ldots|\ldots|$ TATATATTTT ---AGTATAT GAAATTTAC TTTGAGAAAA TTAGAGTGCT AT.-CTAAA.

.T.T..G--.T.T..G--

..T.T.

...АТ....

$\ldots . . .$.

.T.T..G---

AT. TCTAAA.

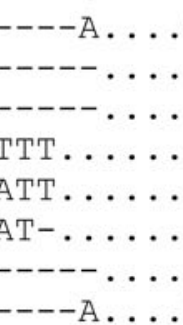

560

570

580

590

600
C. parvum
C. andersoni
C. bovis
C. ryanae
C. hominis
C. suis
C. ubiquitum
C. xiaoi
C. muris
C. parvum
C. andersoni
C. bovis
C. ryanae
C. hominis
C. suis
C. ubiquitum
C. xiaoi
C. muris
C. parvum
C. andersoni
C. bovis
C. ryanae
C. hominis
C. suis
C. ubiquitum
C. xiaoi
C. muris
C. parvum
C. andersoni
C. bovis
C. ryanae
C. hominis
C. suis
C. ubiquitum
C. xiaoi
C. muris

620

630

640

$\ldots|\ldots| \ldots|\ldots| \ldots|\ldots| \ldots|\ldots| \ldots|\ldots| \ldots|\ldots| \ldots|\ldots|$ TTTTTATCTT TCTTATTGGT TCTAAGATAA GAATAATGAT TAATAGGGAC C........

$\ldots \ldots \mathrm{TC}$.

$\ldots \ldots \mathrm{TC}$ G..C..A.G....G.

\section{$\ldots \ldots \ldots$}

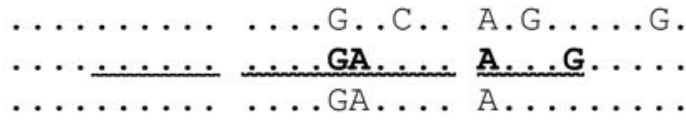

$\ldots \ldots \ldots$

$\ldots \ldots \mathrm{TC}$.

T. . . . . .

. T.......

$\ldots \ldots \ldots \ldots \ldots \ldots$

C...G....

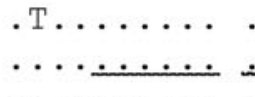

GA.

660

670

680

690

700

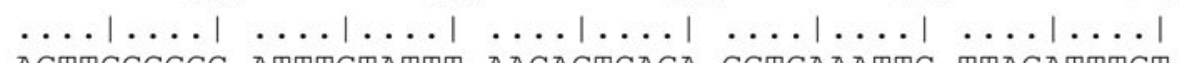
AgTTGGgGGC ATTTGTATTT AACAgTCAgA GGTGAAATTC TTAGATTTGT .

$$
\begin{aligned}
& \cdots \\
& \cdots \\
& \cdots \\
& . \\
& . \\
& .
\end{aligned}
$$

$$
\begin{aligned}
& \\
& \ldots \\
& \text { TAA } \\
& \ldots \\
& \ldots \\
& \ldots \\
& \ldots \\
& \ldots \\
& \ldots
\end{aligned}
$$

Fig. 1. Continued 

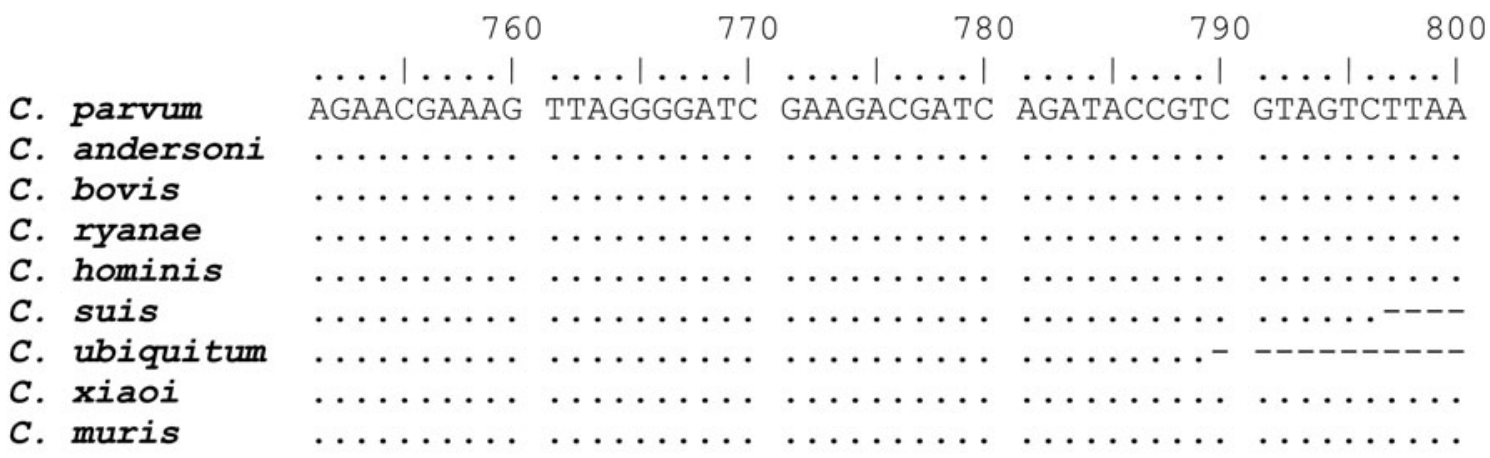

AGAACGAAAG TTAGGGGATC GAAGACGATC AGATACCGTC GTAGTCTTAA

C. andersoni
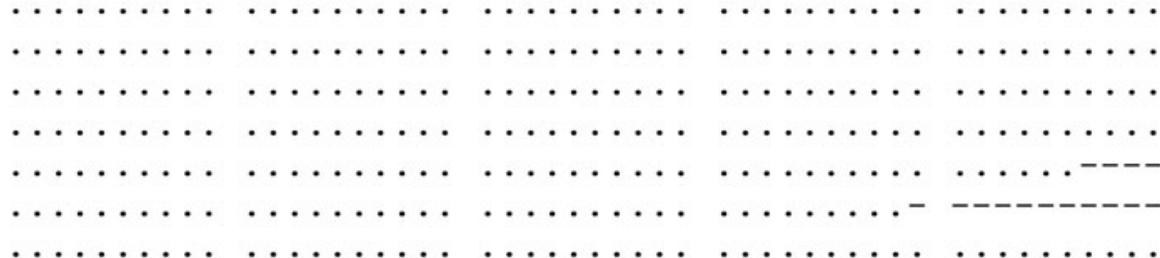

C. muris

C. parvum

C. andersoni

C. bovis

C. ryanae

C. hominis

C. suis

C. ubiquitum

C. xiaoi

C. muris

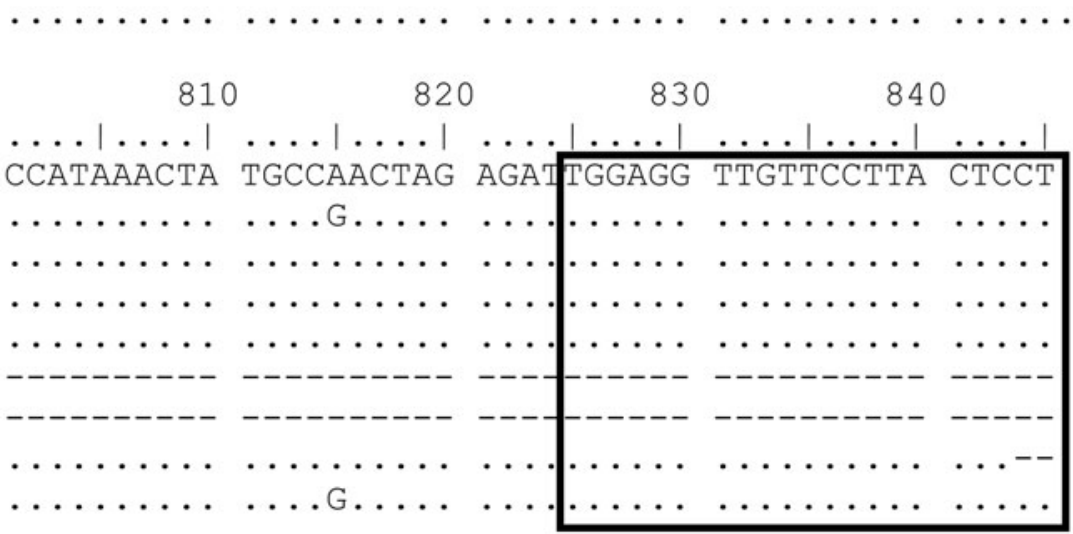

Fig. 1. Continued

the nssm-PCR were compared with sequence data for all of these samples.

\section{Sequence analysis}

To confirm the results of the nssm-PCR when testing field samples, sequence analysis of the $18 \mathrm{~S}$ rRNA amplicon was carried out. Sequencing of PCR products was carried out by GATC (GATC, Germany) using their 'Light-run' service. Roughly $5 \mu \mathrm{L}$ of purified PCR product was sent at a concentration of between 20 and $80 \mathrm{ng} \mu \mathrm{L}$ with 5 $\mu \mathrm{L}$ of the appropriate primer at a concentration of $5 \mathrm{pm} \mu \mathrm{L}$. In some circumstances, instead of the light-run service, unpurified PCR products were sent to GATC from sequencing using their 'Supremerun96' sequencing option which includes PCR purification. With this service $20 \mu \mathrm{L}$ PCR product was sent with a separate $1.5 \mathrm{~mL}$ tube containing $50 \mu \mathrm{L}$ of the appropriate primer at a concentration of $10 \mathrm{pm} \mu \mathrm{L}$. Sequences were analysed using BioEdit Sequence Alignment Software (www.mbio. ncsu.edu/bioedit/bioedit.html) and Chromas Lite (www.technelysium.com).

\section{Sensitivity}

The sensitivity of the nssm-PCR assay was assessed as part of another study (Wells B, Thomson S, Innes E.A and Katzer F Development of a sensitive

Table 1. Primer sequences, size of amplicon and species detected with each primer pair

\begin{tabular}{|c|c|c|c|}
\hline Primer pair & Sequence $5^{\prime}-3^{\prime}$ & $\begin{array}{l}\text { Fragment } \\
\text { size (bp) }\end{array}$ & Species detected \\
\hline AL1687 (EF) AL1691 (ER) & $\begin{array}{l}\text { TTCTAGAGCTAATACATGCG } \\
\text { CCCATTTCTTCGAAACAGGA }\end{array}$ & 1370 & $\begin{array}{l}\text { Genus-specific } \\
\text { external }\end{array}$ \\
\hline AL1598 (IF) AL3032 (IR) & $\begin{array}{l}\text { GAAGGGTTGTATTTATTAGATAAAG } \\
\text { AAGGAGTAAGGAACAACCTCCA }\end{array}$ & 840 & Genus-specific internal \\
\hline CaF AL3032 (IR) & $\begin{array}{l}\text { GCAAATTACCCAATCCTGAC } \\
\text { AAGGAGTAAGGAACAACCTCCA }\end{array}$ & 625 & C. andersoni \\
\hline CrF AL3032 (IR) & $\begin{array}{l}\text { TGTTAATTTTTATATACAATR }{ }^{\mathrm{a}} \text { CTACGG } \\
\text { AAGGAGTAAGGAACAACCTCA }\end{array}$ & 415 & C. ryanae \\
\hline CphF AL3032 (IR) & $\begin{array}{l}\text { AGAGTGCTTAAAGCAGGCATA } \\
\text { AAGGAGTAAGGAACAACCTCCA }\end{array}$ & 305 & C. parvum \\
\hline CbF AL3032 (IR) & $\begin{array}{l}\text { CTTCTTATTCCTTCTAGAATAAAAATG } \\
\text { AAGGAGTAAGGAACAACCTCCA }\end{array}$ & 241 & C. bovis \\
\hline
\end{tabular}

\footnotetext{
a The degenerate base in the C. ryanae primer is shown in bold and underlined.
} 
method to extract Cryptosporidium oocysts from adult cattle fecal samples - submitted). Fecal samples were spiked with C. parvum oocysts, subjected to different concentration steps and DNA extracted. The DNA was then amplified using the nssm-PCR.

\section{RESULTS}

\section{Primer design}

The primer designed for C. parvum will also amplify $C$. hominis and $C$. suis, the $C$. andersoni primer will also amplify $C$. muris and the $C$. bovis primer will amplify $C$. xiaoi as the $18 \mathrm{~S}$ region of these species are very similar (Fig. 1). Cryptosporidium suis and $C$. hominis have only been found very rarely in cattle (Smith et al. 2005; Fayer et al. 2006) and $C$. muris and $C$. xiaoi have yet not been identified in cattle.

\section{Cryptosporidium-specific $18 S$ rRNA PCR}

All 498 samples were tested for the presence of Cryptosporidium parasite DNA using the 18S genus-specific PCR, $4 \cdot 13 \%(n=13)$ of the samples from adult cattle and $60 \cdot 5 \% \quad(n=115)$ of the samples from calves tested positive for Cryptosporidium. These samples were used to test the nssm-PCR.

\section{Individual species PCR}

Amplicons of the expected size for each species $(C$. andersoni, $C$. ryanae, $C$. bovis and $C$. parvum) were obtained when tested in a single species PCR with the species-specific forward primer and the $18 \mathrm{~S}$ rRNA reverse primer. DNA was obtained from samples (confirmed by sequencing) from calves

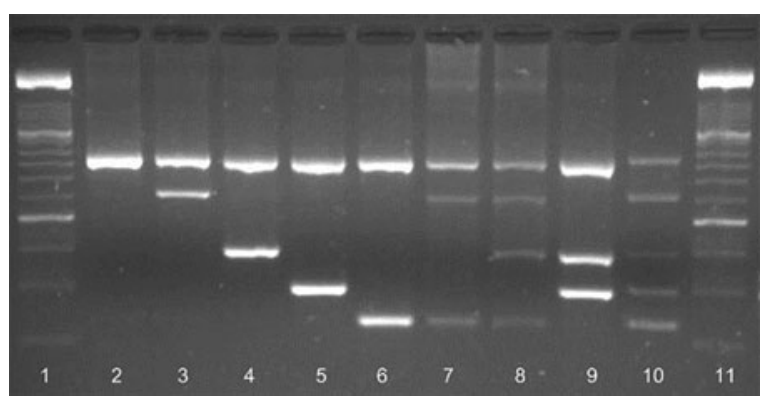

Fig. 2. Amplification of Cryptosporidium spp. on a $2 \%$ agarose gel stained with GelRedTM (Biotum, UK). Lane 1, $100 \mathrm{bp}$ marker XIV (Roche); lane 2, 18S rRNA genusspecific amplicon (840 bp); lane 3, C. andersoni (625 bp); lane 4, C. ryanae (415 bp); lane 5, C. parvum (305 bp); lane 6, C. bovis (241 bp); lane 7, C. andersoni and C. bovis; lane 8, C. andersoni, C. ryanae and C. bovis; lane 9, C. ryanae and $C$. parvum; lane 10, nssm-PCR molecular marker; lane 11, 100 bp marker XIV (Roche). Amplicons in lanes 2-6 were produced using cloned DNA and lanes 7-9 were from naturally infected cattle. aged $<1$ week (C. parvum DNA), calves aged 3 months $(C$. bovis and $C$. ryanae DNA) and an adult cow (C. andersoni DNA).

\section{Nested species-specific multiplex PCR}

Amplicons of the expected size for each of the four species (C. andersoni, C. ryanae, $C$. parvum and $C$. bovis - individually and mixed combinations) were obtained using the nssm-PCR assay (Fig. 2) from a variety of cattle samples collected from calves aged $7-14$ days regardless of clinical signs $(n=116)$, all calves suffering from diarrhoea regardless of age $(n=74)$, adult cattle pre- and post-calving $(n=177$ and $n=120$, respectively) and other adult cows $(n=11)$. The expected amplicons for all four cattle species $[C$. parvum $(n=92), C$. andersoni $(n=1), C$. ryanae (only in mixed infections) and $C$. bovis $(n=$ 13)] were observed as were various combinations of mixed infections (Fig. 2).

Occasionally an additional non-specific band appeared with the amplicon for $C$. parvum but of a different size to the species-specific bands. The results of the nssm-PCR were compared with sequence data for the $18 \mathrm{~S}$ rRNA gene of these samples. In all cases, the single species of Cryptosporidium identified by nssm-PCR matched the species identified by sequencing. In addition, the nssm-PCR identified mixed infections in 14 of the samples examined. The mixed infections included; C. bovis, C. parvum and C. ryanae $(n=$ $1), C$. ryanae and $C$. bovis $(n=5), C$. parvum and $C$. bovis $(n=1), C$. parvum and C. ryanae $(n=1)$ and $C$. andersoni and $C$. ryanae $(n=6)$. These mixed infections were not identified by sequencing. Eight of the samples failed to amplify using the nssm-PCR.

\section{Sensitivity}

The sensitivity of the nssm-PCR when tested on fecal samples spiked with $C$. parvum oocysts was 5 opg (oocysts per gram of feces) (Table 2).

\section{DISCUSSION}

In this paper, we describe the development of a multiplex PCR test, which allows the discrimination

Table 2. Sensitivity of nssm-PCR when tested on DNA extracted from feces spiked with $0,5,10,100$ or 1000 C. parvum oocysts per gram

\begin{tabular}{lllll}
\hline \hline 0 & 5 & 10 & 100 & 1000 \\
- & ++ & +++ & +++ & +++ \\
\hline \hline
\end{tabular}

- , negative; + , positive $1 / 3 \mathrm{PCRs} ;++$, positive $2 / 3 \mathrm{PCRs}$; +++ , positive $3 / 3$ PCRs. 
of four of the most commonly detected Cryptosporidium species of cattle (one of which poses a risk to human health). The test allows the differentiation of these species by generating distinct amplicons of the $18 \mathrm{~S}$ rRNA gene: $C$. andersoni $(625 \mathrm{bp})$, $C$. ryanae $(415 \mathrm{bp}), C$. parvum $(305 \mathrm{bp})$ and $C$. bovis (241 bp) in addition to a Cryptosporidium genus-specific amplicon (840 bp). Currently, the most popular method of species identification in Cryptosporidium-positive samples is gene sequencing of the $18 \mathrm{~S}$ rRNA gene. This method is very sensitive and can detect a large range of Cryptosporidium species and genotypes, but is limited when mixed infections occur as it has been shown that in a mixed sample either the dominant Cryptosporidium species is preferentially amplified by PCR meaning that only one species will be identifiable by sequencing or the sequence is unreadable (Santin et al. 2004; Cama et al. 2006; Silverlas et al. 2010). PCR amplification followed by sequence analysis is also more costly and time-consuming than multiplex PCR. The multiplex PCR described here allows the detection of mixed infections of up to four different common cattle species of Cryptosporidium and is an excellent method for detecting underlying infections of zoonotic $C$. parvum in calves which may have higher burdens of the less pathogenic Cryptosporidium species. This has very important implications for human health and the new test has also proven useful when determining which calves are most suitable for use in calf handing classes at a local veterinary school to reduce the risk of Cryptosporidium infection to students. It has also been used to identify the species of Cryptosporidium present in feces from adult cattle and their calves as part of a whole catchment study in the North East of Scotland (Wells et al. 2015).

In addition to the easy and quick visual differentiation of the four common cattle species other species of Cryptosporidium may be identified by sequencing of the $18 \mathrm{~S}$ rRNA gene when a fragment responding to the Cryptosporidium genus-specific amplicon $(840 \mathrm{bp})$ is amplified with no speciesspecific amplicons. At present, using this method, it is not possible to distinguish between $C$. parvum and $C$. hominis however, as $C$. hominis has rarely been detected in cattle (Smith et al. 2005; Park et al. 2006) there is, at this stage, no real need to distinguish between these two species.

The advantages of the PCR protocol described here over other methods used for species discrimination (PCR-RFLP, sequencing) are: (1) the ability to detect mixed infections, which may be missed by other methods; (2) the ability to identify other Cryptosporidium species by further sequencing when none of the 'cattle species' are detected as other species of Cryptosporidium may be a risk to other animals or humans; (3) the ability to test large numbers of samples quickly and easily; and
(4) the ability to detect subclinical infections of the zoonotic species C. parvum in animals shedding high numbers of the non-zoonotic species.

\section{FINANCIAL SUPPORT}

The authors would like to thank AHDB, EBLEX, HCC, QMS, DairyCo, BBSRC for funding awarded to Sarah Thomson and The Scottish Government for funding awarded to Elisabeth Innes and Frank Katzer.

\section{REFERENCES}

Brook, E., Hart, C. A., French, N. and Christley, R. (2008). Prevalence and risk factors for Cryptosporidium spp. infection in young calves. Veterinary Parasitology 152, 46-52.

Cama, V., Gilman, R. H., Vivar, A., Ticona, E., Ortega, Y., Bern, C. and Xiao, L. (2006). Mixed Cryptosporidium infections and HIV. Emerging Infectious Diseases 12, 1025-1028.

Chalmers, R. M. and Katzer, F. (2013). Looking for Cryptosporidium: the application of advances in detection and diagnosis. Trends in Parasitology 29, 237-251.

Clark, D. P. and Sears, C. L. (1996). The pathogenesis of cryptosporidiosis. Parasitology Today 12, 221-225.

De Graaf, D. C., Vanopdenbosch, E., Ortega-Mora, L. M., Abbassi, H. and Peeters, J. E. (1999). A review of the importance of cryptosporidiosis in farm animals. International fournal of Parasitology 29, 1269-1287.

Fayer, R. (2010). Taxonomy and species delimitation in Cryptosporidium. Experimental Parasitology 124, 90-97.

Fayer, R., Santin, M., Trout, J. M. and Greiner, E. (2006). Prevalence of species and genotypes of Cryptosporidium found in 1-2-year-old dairy cattle in the eastern United States. Veterinary Parasitology 135, 105-112.

Katzer, F., Canton, G., Burrells, A., Palarea-Albaladejo, J., Horton, B., Bartley, P. M., Pang, Y., Chianini, F., Innes, E. A. and Benavides, J. (2014). Immunization of lambs with the S48 strain of Toxoplasma gondii reduces tissue cyst burden following oral challenge with a complete strain of the parasite. Veterinary Parasitology 205, 46-56.

Klein, P., Kleinova, T., Volek, Z. and Simunek, J. (2008). Effect of Cryptosporidium parvum infection on the absorptive capacity and paracellular permeability of the small intestine in neonatal calves. Veterinary Parasitology 152, 53-59.

Lindergard, G., Nydam, D. V., Wade, S. E., Schaaf, S. L. and Mohammed, H. O. (2003). A novel multiplex polymerase chain reaction approach for detection of four human infective Cryptosporidium isolates: Cryptosporidium parvum, types $\mathrm{H}$ and $\mathrm{C}$, Cryptosporidium canis, and Cryptosporidium felis in fecal and soil samples. Fournal of Veterinary Diagnostic Investigation 15, 262-267.

Park, J. H., Guk, S. M., Han, E. T., Shin, E. H., Kim, J. L. and Chai, J. Y. (2006). Genotype analysis of Cryptosporidium spp. prevalent in a rural village in Hwasun-Gun, Republic of Korea. Korean fournal of Parasitology 44, 27-33.

Patel, S., Pedraza-Diaz, S. and McLauchlin, J. (1999). The identification of Cryptosporidium species and Cryptosporidium parvum directly from whole faeces by analysis of a multiplex PCR of the $18 \mathrm{~S}$ rRNA gene and by PCR/RFLP of the Cryptosporidium outer wall protein (COWP) gene. International Fournal for Parasitology 29, 1241-1247.

Ryan, U., Fayer, R. and Xiao, L. (2014). Cryptosporidium species in humans and animals: current understanding and research needs. Parasitology 141, 1667-1685.

Santin, M. and Zarlenga, D. S. (2009). A multiplex polymerase chain reaction assay to simultaneously distinguish Cryptosporidium species of veterinary and public health concern in cattle. Veterinary Parasitology 166, 32-37.

Santin, M., Trout, J. M., Xiao, L., Zhou, L., Greiner, E. and Fayer, R. (2004). Prevalence and age-related variation of Cryptosporidium species and genotypes in dairy calves. Veterinary Parasitology 122, 103-117.

Silverlas, C., Emanuelson, U., de, V. K. and Bjorkman, C. (2009). Prevalence and associated management factors of Cryptosporidium shedding in 50 Swedish dairy herds. Preventative Veterinary Medicine 90, 242-253.

Silverlas, C., Naslund, K., Bjorkman, C. and Mattsson, J. G. (2010). Molecular characterisation of Cryptosporidium isolates from Swedish dairy cattle in relation to age, diarrhoea and region. Veterinary Parasitology 169, 289-295. 
Smith, H. V., Nichols, R. A., Mallon, M., MacLeod, A., Tait, A., Reilly, W. J., Browning, L. M., Gray, D., Reid, S. W. and

Wastling, J. M. (2005). Natural Cryptosporidium hominis infections in Scottish cattle. Veterinary Record 156, 710-711.

Tzipori, S. and Ward, H. (2002). Cryptosporidiosis: biology, pathogenesis and disease. Microbes and Infection 4, 1047-1058.

Wells, B., Shaw, H., Hotchkiss, E., Gilray, J., Ayton, R., Green, J., Katzer, F., Wells, A. and Innes, E. (2015). Prevalence, species identification and genotyping Cryptosporidium from livestock and deer in a catchment in the Cairngorms with a history of a contaminated public water supply. Parasites and Vectors 8, 66.

Wyatt, C. R., Riggs, M. W. and Fayer, R. (2010). Cryptosporidiosis in neonatal calves. Veterinary Clinics of North America - Food Animal Practice 26, 89-103,

Xiao, L., Alderisio, K., Limor, J., Royer, M. and Lal, A. A. (2000). Identification of species and sources of Cryptosporidium oocysts in storm waters with a small-subunit rRNA-based diagnostic and genotyping tool. Applied and Environmental Microbiology 66, 5492-5498. 